\title{
The Evolutionary Law of Information in the Material World: Five Elements Theory
}

\author{
Ruiyu Zhu ${ }^{* 1}$ \\ ${ }^{1}$ Jiangnan University, Wuxi, P.R. China.
}

\section{Keywords}

wuxing, five elements, laws of evolution, chinese philosophy

\begin{abstract}
The Five Elements theory (Wuxing) is the philosophical foundation of traditional Chinese medicine and culture. 'Metal', 'Water', 'Wood', 'Fire', and 'Earth' are the five basic elements of the Five Elements theory. However, due to its complexity, people's understanding of the theory has remained superficial, giving rise to great differences in understanding. Scientists often regard the Five Elements theory as unscientific. At the same time, enthusiasts of traditional Chinese culture often overuse it and reach specious conclusions, posing a challenge to rational understanding by the public. This study proposes that in nature-a dynamic and complex system-evolution determines the existence of the law of matter, and the law of matter exists to better perform evolution. Yinyang and Wuxing are the laws of evolution. Using scientific logic, it is also shown that Wuxing is the most basic movement mode of complex dynamic systems, reflecting the basic dynamic mechanism of complex systems' responses to environmental information. This study further proposes that 'Metal' is the control center of the entire system and represents the response, processing, and distribution of internal information commands. 'Water' represents the process of internalizing energy and the inflow of basic materials. 'Wood' represents the process of forming a high-level substance based on information. 'Fire' represents the realization of the function of advanced matter and satisfying the information needs. Finally, 'Earth' represents the process of returning advanced substances to basic substances and the feedback from material world to the original information. In fact, any complex phenomenon is nothing but the evolution of information in the material world, revealing the scientific nature of the Five Elements can provide new ideas for the human cognition of complex phenomena and provide a new perspective for solving complex system problems.
\end{abstract}

In the face of nature, looking for objective laws embedded in nature, Western scientists tend to look at the material composition, determine the structure, and further study the interactions between different substances. In the ancient East, meanwhile, the sages focused on examining the movement of things, and thus, proposed the theories of Yinyang and Wuxing (the Five Elements), which provide the theoretical basis for traditional Chinese medicine and culture. Between these two different tendencies, there is no doubt that Western science has produced a more objective understanding of nature and has greatly furthered the development of the entire world. However, since its development thousands of years ago, the traditional Chinese mode of thinking has stagnated and has gradually been abandoned over the past 100 years. As science is highly developed today, in the face of complex issues-especially those related to life sciences and various human events-we have become lost and confused. It is important, therefore, to consider whether the Yinyang and Wuxing of ancient oriental thought might have essential

\footnotetext{
$*$ This is a working document which has not been peer reviewed yet. Please address any comment or remark to ry_zhu@hotmailcom or ry_zhu@sina.com.
} 
scientific connotations. Such an understanding could provide a new perspective for revealing the mysteries of nature and solving problems related to complexity.

To this end, we need to revisit the overall understanding of nature in contemporary science. According to the current scientific knowledge, the Big Bang theory may account for the origin of the universe. Singularity refers to a point of infinite mass and infinitesimal volume. The explosion of the singularity further formed basic particles and then further formed atoms, molecules, macromolecules, single-cell systems, multicell systems, people, society, and so on. When considering such things, we often focus on their composition and interactions while ignoring the driving force in the process of movement from elementary particles to complex individuals and society; this force is evolution. We can see that there are different objective laws at different levels of substance, which are not controlled by human beings. These laws only arise when the corresponding material basis appears, and new laws are still emerging. Obviously, for the entire process - from the singularity to the evolution of human society-it is evolution that determines the existence of follow-up laws, and the purpose of the follow-up laws is to better perform in-depth evolution. An obvious example is that human society is constantly making various rules, the purpose of which is to integrate the society as a whole at a higher level so it can move forward. Of course, sometimes degeneration will occur, but evolution is the general trend. As another example, various biological laws at the cell level are developed after the emergence of cells, along with the cooperation of various types of cells. Adaptive evolution allows some interactions to appear in a steady state, which, in turn, allows some biological laws to exist more stably. For example, the law of DNA replication occurs after DNA, polymerase, and other auxiliary components appear and collaborate. It continues to deepen in the evolutionary path of prokaryotic and eukaryotic cells, thereby enriching its regulatory mechanisms and making biological forms more colorful. For yet another example, we humans developed and evolved from fertilized eggs. The process from fertilized egg to human development is a naturally driven process that does not require the active participation of our consciousness. We can see the presence of some cytokines and their interactions only after the formation of tissues and related cells; some of them merely appear in specific cell states. The purpose of various cellular operating mechanisms is to enable cells to better evolve into fully functional individuals and get along well with the environment.

Therefore, evolution is the foundation and root of all of material laws. We can imagine that without the explosion from the singularity, all of the laws of matter would not have been able to appear. Without the appearance of cells caused by evolution, biological laws would not have been able to appear. The emergence of various laws allows evolution to continue and develop in more colorful directions, which is particularly obvious in various phenomena in human society. In fact, this evolutionary dynamic has been at work, and everything is inevitably affected by this driving force. Are there laws in the process of evolution? Yinyang and Wuxing in traditional Chinese philosophy may represent basic evolutionary laws. The nature of Yin and Yang and their role in evolution are relatively easy to understand. It is, moreover, easy for people to establish related concepts, which have been discussed in many articles. As for Wuxing, there is still much confusion. In this article, I will discuss the scientific nature of the Five Elements. 


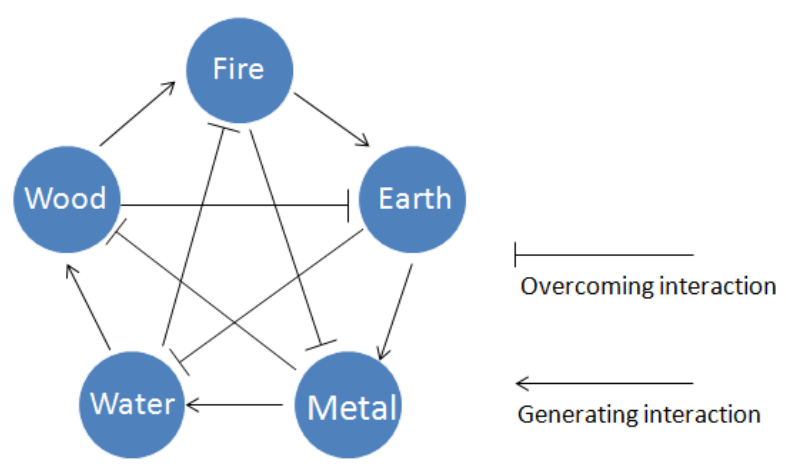

Fig.1 Diagram of the interactions between the Five Elements

The Five Elements doctrine is the most important ideological doctrine in ancient Chinese philosophy after the Yinyang doctrine. Together with Yinyang theory, Wuxing forms the basis of the ancient Chinese scientific theoretical system. The main ideological content of the Five Elements theory can be described as follows: 'The Five Elements theory believes that everything in the universe is composed of five basic elements: wood, fire, earth, metal, and water. The development and changes of all kinds of things and phenomena are the result of the continuous movement and interaction of these five elements. The order of the movement of all things in the world is subject to the unified control of the Five Elements'.

Of course, from the original concept of the Five Elements, we can hardly perceive a scientific connotation. However, if we can change the perspective and treat these Five Elements as abstract dynamic elements, a relatively reasonable explanation may be obtained. Everything is in motion. In a dynamic and complex system, the most basic interaction among the components is the positive or negative regulation of other components, as well as being positively or negatively regulated by other components. The independent, abstract basic elements in a complex system fall under these four basic forces; thus, the system as a whole obtains a relatively steady state and existence. If each function points to a different element, the basic unit of the system is the Five Elements. In such a complex system composed of five independent components, each component can obtain the four most basic interaction forces, and each component is in the 'equivalent state'. If there are less than five, there will be more than one element that lacks the function of positive or negative regulation. Thus, the element is in an inactive or uncontrolled state, which poses a huge challenge to the stability of the system. If there are more than five, there must be some components with overlapping functions, which cannot be defined as basic components.

Obviously, these five basic elements must have a positive control activation link chain, thus forming a closed-loop linear activation loop. Due to the lack of negative regulation, the loop is unstable. Regarding the negative adjustment part of the Five Elements system, we can also discover its necessity through logical analysis. First, each element must necessarily have the negative regulation of other elements. If an independent element only has positive regulation and no negative regulation of the system itself, then the overpresentation of this independent element will inevitably have a destructive effect on the stability of the entire system. Therefore, any independent element in a system will necessarily have both positive and negative regulation for other elements. Second, such negative regulation can only occur in the second or third element downstream of the positive regulation chain. Obviously, the second component downstream of negative regulation is a steady-state mode. If negative regulation occurs in the 
third element, the element will down-regulate the negative regulation element of the downstream-activated element, thereby suppressing the negative regulation of the downstream activation element, which will eventually cause the system to collapse. Therefore, the Five Elements theory is the basic mechanism of cybernetics for complex systems, and it has a strong scientific and logical foundation. These five independent elements can be abstract things such as the functions or movement of things.
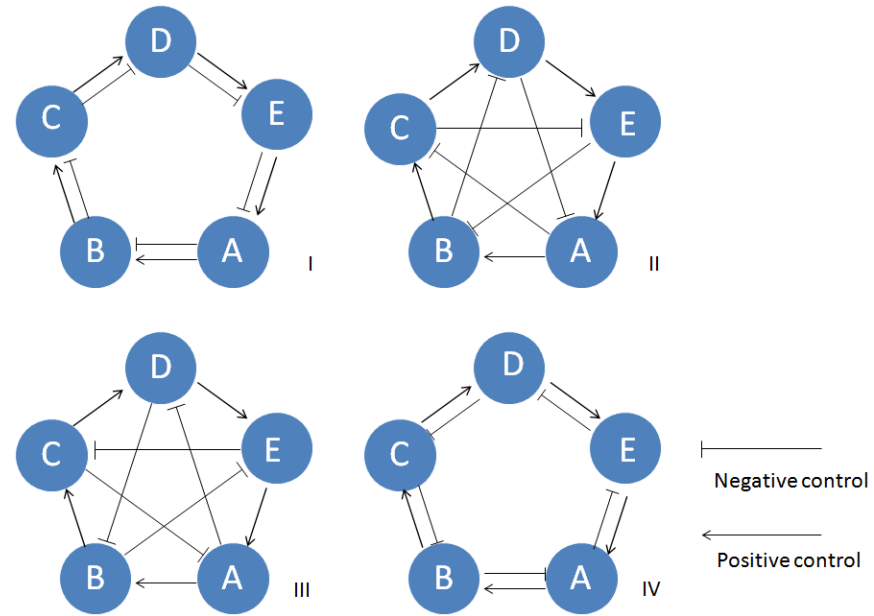

Fig.2 Possible negative feedback modes among the Five Elements

Considering that the Five Elements theory is the basic law of a dynamic complex system, what do the basic components represent? Under modern scientific thought, matter, energy, and information are undoubtedly the most basic abstract elements in the objective world. On this basis, combined with the knowledge of traditional Chinese culture, further analysis of these Five Elements can provide the following understanding: 'Metal' is the control center of the entire system, representing the inflow of environmental information, the processing of information, and the distribution of internal information. 'Water' represents the internalization of energy and the inflow of basic substances. 'Wood' represents a combination of basic substances based on information to form advanced substances. 'Fire' represents the externalization of energy, the realization of the function of advanced matter and satisfying the information needs. Finally, 'Earth' represents the process of returning advanced substances to basic substances and the feedback from material world to the original information. We need to understand that these structures have a logical basis. In addition, the process described by this theory is very consistent with objective reality; this should represent the basic objective laws possessed by the dynamic system itself. 


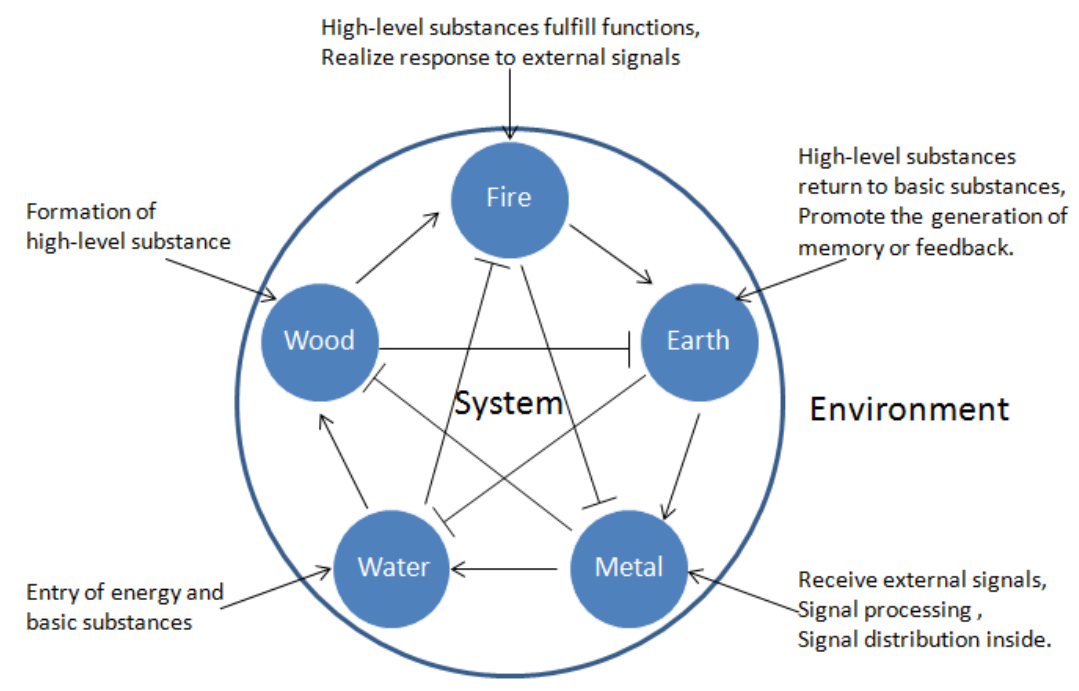

Fig. 3 The scientific connotation of the Five Elements

Is this system feasible in reality? The Five Elements theory is one of the theoretical foundations of traditional Chinese medicine. In traditional Chinese medicine, metal represents the lungs, water the kidneys, wood the liver, fire the heart, and soil the spleen. The lungs, kidneys, liver, heart, and spleen in traditional Chinese medicine are different from those in Western medicine. In traditional Chinese medicine, each organ represents a type of system, which has not been fully explained. To facilitate the discussion, I will analyze the organ as representative of a system. Then, we will see that the scientific connotation of the Five Elements described earlier matches the corresponding functional designations of traditional Chinese medicine quite well. 'Water', representing the accumulation of energy and basic substances, precisely coincides with the function of the kidney. 'Wood', representing the production of high-level substances, coincides with the function of the liver. 'Fire' represents the release of energy, and the appearance of that function coincides with the heart. 'Earth' represents the return or degradation of high-level substances, coinciding with the function of the spleen. The explanation of 'Metal' is a bit more complicated. How to understand the interrelationships between 'Metal', lungs, and information? 'Metal' represents information or, in a sense, the regulation of subsystems by higher level systems or the interaction of environments with subsystems. The main function of the lung is to obtain oxygen from the environment and release carbon dioxide. In a sense, it is a kind of regulation of the higher level environmental system of human life and the controlling element of human energy production. Therefore, the connection between 'Metal', information, and lung seems to be reasonable. From this, we can suggest that this information is also a law to some extent. The law itself is a method of the advanced system-control subsystem, and since it is not determined by the running system, it is very powerful. How similar are its characteristics to metals? Indeed, we accumulated energy and basic matter and further formed a higher level of matter or life.

As another example, we can use the Five Elements theory to analyze the entire process of cell information display. DNA is the source of cellular information. In response to environmental signals, the transcriptional activation of related information may represent 'Metal'. The intake and transport of energy and basic substances related to transcription and translation can represent 'Water'. RNA transcription and subsequent protein translation can represent 'Wood'. Functional RNAs and proteins are transported to their functional sites, which can represent 'Fire', 
and functional RNA and protein are degraded after completing their functions, which can represent 'Earth'. Whether such a process will form a memory effect depends on the epigenetic modification of DNA. Similarly, this path from information to material presentation to information feedback can be one of continuous information accumulation. Thus, the Five Elements system shows the evolution process from information to matter and then from matter to information.

The Five Elements system can also be understood in terms of events in our lives. To do something, a person must first memorize the relevant information to analyze the reception of external signals and the issuance of related internal commands ('Metal'). Then, the person must obtain funds and basic materials related to the completion of the activity ('Water'). Next, it is necessary to further construct the material basis of the event ('Wood'), carry out the event and realize its function ('Fire'), and handle and return the related substances after the event ('Earth'). Finally, the person creates feedback ('Metal').

When signals from the environment concern the new birth, maturity, and decay of a system, the different stages of the life process of the system must be in a typical period of one of the five basic elements, thus showing the corresponding state. Similarly, the state of each person, individual, or organization can be divided according to these five states. At the macro-level, the interaction between these five states is sometimes less obvious. The motion law of the Five Elements can help us dynamically understand complex systems (e.g., cellular dynamic signal networks and complex social events), along with holistic ways of thinking and understanding. The application of the Five Elements rule to specific scientific and social events also needs to be based on specific circumstances, and the activation and suppression modes need to be supported by real evidence. In any case, the Five Elements theory can provide us with a new focus, a more comprehensive and systematic way of thinking, and a paradigm that differs from the current thinking dominated by linear logic.

Based on the above analysis, we can see that for complex systems, the Five Elements theory has powerful theoretical and practical properties. Of course, the functions of mutual promotion and inhibition can be described using other terms, such as mutual support and mutual restraint, harmful, and beneficial. If we can conduct more in-depth research and practice related to the Five Elements theory, we might find that this cognitive point of view is not so simple, and that it has an important value and significance for thinking about and solving various complex problems in real life.

\section{References}

1. Gore,J., van Oudenaarden, A. The yin and yang of nature. Nature,2009 457, 271-272.

2. Wang Y. Wuxing Theory and Ethics for Health Care in China. Tangdu Journal, 2006, 022(004):38-40. 